\title{
Study on the Mixed-Mode HPLC Separation of Shuang-Huang-Lian and Xue-Bi-Jing Injections Based on an Ionic Liquid Column
}

\author{
Aihong Yang, Shun Zhang, Lei Zhang, Jingjing Zhong and Xiaodi Kou* \\ School of Chinese Materia Medica, Tianjin University of Traditional Chinese Medicine, \\ 300193 Tianjin, P. R. China
}

\begin{abstract}
Both Shuang-Huang-Lian and Xue-Bi-Jing injections are clinically proven efficient and low cost medicines. However, the studies of their chromatographic separation are limited. In this study, a column based on ionic liquid stationary phase was applied for the first time for the analysis of these two injections. For Shuang-Huang-Lian injection, U-shaped plots of retention factors $v s$. mobile phase constitutions were obtained. This unique kind of separation behavior allowed a flexible adjustment of retention by tuning the mobile phase constituents. Then acids group was analyzed with hydrophilic chromatography mode, while glycosides group was analyzed with reversed phase chromatography mode. Similarly, constituents in Xue-Bi-Jing injection were analyzed with mixed mode high performance liquid chromatography (HPLC). Higher, medium and lower polarity groups were analyzed with hydrophilic interaction, reversed phase and normal phase liquid chromatography modes, respectively. Results from this work provided valuable information for the separation of complicated systems such as traditional Chinese medicine injections.
\end{abstract}

Keywords: ionic liquid stationary phase, mixed-mode chromatography, Shuang-Huang-Lian injection, Xue-Bi-Jing injection

\section{Introduction}

The formulations of traditional Chinese medicines (TCMs) are becoming increasingly popular worldwide, mainly because of their high efficiency and low cost. However, TCM formulations are usually based on a whole plant and/or combination of several herbs, which contain more than dozens to hundreds of components. ${ }^{1}$ Therefore, in order to ensure both the safety and efficacy of TCM formulations, it is necessary to establish simple and effective methods to determine as many constituents as possible. ${ }^{2,3}$ Liquid chromatography (LC) has been applied as a rapid and economic method for the determination of multi-component in TCMs. ${ }^{4}$ Among the liquid chromatography analytical methods, high performance liquid chromatography (HPLC) is the most popular one, due to its easy operation, wide suitability and high accuracy for the qualitative and quantitative analysis of TCMs.$^{5-7}$ However, in practice, some factors, such as efficiency, selectivity and sensitivity have been found to affect the analysis of complicated systems with HPLC. ${ }^{8}$

*e-mail: xiaodikou@tjutcm.edu.cn
Chromatographic stationary phase is the core of HPLC system. Therefore, the development of novel stationary phases with high separation efficiency and excellent selectivity has always been the focus point of improving the separating ability of HPLC. Accordingly, in this work novel ionic liquid (IL) bonded stationary phase has been studied. IL is a kind of novel material composed of ions and at the same time remains in liquid state at a temperature below $100{ }^{\circ} \mathrm{C} .{ }^{9}$ ILs have demonstrated the versatility and tuneability in separation science. ${ }^{10}$ Many types of ILs were immobilized on a support like silica or monolith as stationary phases for liquid chromatography. ${ }^{11,12}$ Due to the designable character of the IL molecule, multi interactions between the IL stationary phases and the analytes can be shown, including hydrophobic, hydrophilic, hydrogen bond, anion exchange, $\pi-\pi$, and dipole-dipole interactions. ${ }^{11,12}$ Therefore, they could be used in different chromatographic modes including ion-exchange, reversedphase liquid chromatography (RPLC), ${ }^{13-15}$ normalphase liquid chromatography (NPLC) and hydrophilic interaction liquid chromatography (HILIC) ${ }^{16-18}$ to separate various classes of compounds. When the stationary phase interacts with solutes through more than one interaction mode, it is called a mixed-mode chromatography. ${ }^{19}$ 
One mixed-mode column can be used to replace two or more single-mode columns, offering more selectivity for simultaneous separation of diverse compounds. Recently, two research groups have used ILs columns for mixedmode chromatography. Zhang and co-workers ${ }^{20}$ synthesized calixarene ionic liquid modified silica gel stationary phase. They evaluated its chromatographic performance with RPLC, HILIC and anion exchange modes. In Qiu and co-workers' report, ${ }^{21}$ 2-methylimidazolium-functionalized silica stationary phase was prepared and further used for hydrophilic interaction and anion-exchange mixed-mode chromatography.

We expect mixed-mode chromatography will be suitable for the analysis of complex systems, such as TCM injections. So in this work, we prepared columns with methyl imidazole ionic liquid bonded silica as the stationary phase and applied them for the analysis of Xue-Bi-Jing (XBJ) and Shuang-Huang-Lian (SHL) injections. We also did a detailed study on tuning its mixed-mode separation by adjusting chromatographic conditions. There have been some literature reports about the quality control of XBJ and SHL injections. And because of the complicated nature of TCM formulations, the separation method should be carefully selected based on the analytes. In this work we took the analysis of XBJ and SHL injections as the research objects of the mixed mode separation.

SHL injection is a composite formula of TCM extracted from three herbs: Radix Scutellariae, Scutellaria baicalensis and Fructus Forsythiae. ${ }^{22}$ Clinically, it is commonly used to treat acute upper respiratory tract infections caused by viruses or bacterial infection. ${ }^{23}$ Despite its high clinical efficacy, applications of SHL injection have been reported with severe adverse drug reactions majorly manifested as allergic reactions caused by chlorogenic acids and baicalin..$^{24,25}$ In order to ensure the safety of the SHL injection, several research groups have reported studies focused on the development of sensitive analytical methods to identify the real allergenic constituents in SHL injection. He and co-workers ${ }^{26}$ built a two-dimensional analytical system by combining cell membrane chromatography with liquid chromatography tandem mass spectrometry (LC/MS) and baicalin was identified as the potential allergenic component. Chen and co-workers ${ }^{27}$ did a thorough study focused on the overall constituents by HPLC-DAD-ESI-IT-TOF-MS method and they identified 125 multifarious constituents in SHL powder injection. Kotani and co-workers ${ }^{28}$ developed a novel three-channel isocratic elution liquid chromatography with electrochemical detection system for the simultaneous determination of various bioactive compounds in SHL injection.
XBJ injection, a traditional Chinese five-herb prescription consisting of Flos Carthami, Radix Paeoniae Rubra, Rhizoma Chuanxiong, Radix Salviae miltiorrhizae and Radix Angelicae sinensis, can effectively promote blood circulation and microcirculation. Clinically, XBJ can significantly shorten the course of sepsis, lower the temperature and improve breathlessness. ${ }^{29,30}$ In order to guarantee the efficacy of XBJ injection, some research has been done on both qualitative and quantitative analysis of the chemical composition of XBJ. ${ }^{31-34}$ Most of these studies have been focused on better quality control of XBJ injection through different detection methods. For example, Bai group applied the time-of-flight (TOF) MS or bioactivity-integrated quadrupole (Q)/TOF methods; ${ }^{31,32}$ Zheng and co-workers ${ }^{33}$ developed the method of electrochemical detection with $\mathrm{IL}$ and nanogold particles as additives; while the group of Zhang and co-workers ${ }^{34}$ operated the detection with high resolution ultra HPLC hybrid quadruple-orbitrap mass spectrometry (UHPLC-Q-Orbitrap MS).

In summary, all of the chromatographic separations of SHL injection were done with columns of traditional $\mathrm{C}_{18}$ stationary phase. In this work, we studied the chromatographic separation of SHL injection with an IL column. We expect the introduction of a new kind of stationary phase material may contribute to a more sensitive chromatographic separation, which is believed by many researchers to be a crucial part for the analysis of TCM formulations. In our study, we did some preliminary research about the chromatography separation of SHL and XBJ injections based on column of novel IL bonded stationary phase with readily available HPLC-UV instrument. We expect this work will help to improve the quality control of TCM formulations from a new aspect.

\section{Experimental}

\section{Materials and reagents}

Chromatography grade acetonitrile $(\mathrm{ACN})$ and methanol $(\mathrm{MeOH})$ were purchased from Fisher Scientific (USA). Ultra-pure water was purchased from Watsons Group (China). Toluene, hydrochloric acid and phosphoric acid were purchased from Concord Chemical (China). 3-Chloropropyltrimethoxysilane (98\%) and $\mathrm{N}$-methylimidazolium (99\%) were obtained from Energy Chemical (China). Spherical porous silica particles (diameter $5 \mu \mathrm{m}$, pore size $100 \AA$, specific surface area $300 \mathrm{~m}^{2} \times \mathrm{g}^{-1}$ ) were purchased from BoRui Bonded Chromatographic Techniques Company (China). All of the standards for chromatographic analysis were purchased from Aladdin (China). 
Instrumentation

Elemental analysis was performed on a Vario EL Cube CHONS elementary analyzer (Germany). The Fourier transform infrared (FTIR) spectra were collected from a Thermo Nicolet Nexus 870 FT-IR spectrophotometer (USA). Solid nuclear magnetic resonance (NMR) spectra were collected from a Bruker, AVANCE III $400 \mathrm{MHz}$ (USA).

Synthesis of the $N$-methylimidazolium-grafted silica stationary phase

The $N$-methylimidazolium-grafted silica was synthesized as following with a modified literature method. ${ }^{35}$

First, the silica was immersed in hydrochloric acid for $24 \mathrm{~h}$ and then washed with deionized water and dried under vacuum for $8 \mathrm{~h}$ at $120{ }^{\circ} \mathrm{C}$. The activated silica $(5.0 \mathrm{~g})$ was suspended in $60 \mathrm{~mL}$ of dry toluene and then an excess of 3-chloropropyltrimethoxysilane $(5.0 \mathrm{~g})$ was added, followed by adding $0.5 \mathrm{~mL}$ of hydrochloric acid as a catalyst. The suspension was stirred and refluxed for $24 \mathrm{~h}$. After the reaction was stopped, the reaction mixture was transferred to a vacuum glass filter and washed with ethanol $(3 \times 30 \mathrm{~mL})$ and water $(3 \times 30 \mathrm{~mL})$ in turn and at last ethanol $(30 \mathrm{~mL})$. Chloropropyl-bonded silica (ClPr-Sil) was dried under vacuum at $80{ }^{\circ} \mathrm{C}$ for $12 \mathrm{~h}$ before the next reaction step. Finally, $4.8 \mathrm{~g}$ ClPr-Sil were placed in a $250 \mathrm{~mL}$ reaction flask containing $82 \mathrm{~mL}$ toluene, and then $4.8 \mathrm{~g} \mathrm{~N}$-methylimidazolium were added. The mixture was stirred at $110{ }^{\circ} \mathrm{C}$ for $48 \mathrm{~h}$. The reaction mixture was filtered and washed with ethanol and water. The obtained methylimidazol bonded silicon (Mim-Sil) was dried under vacuum at $80^{\circ} \mathrm{C}$ for $10 \mathrm{~h}$, prior to characterizations.

\section{Column packing}

The resulting modified silica materials were packed into stainless-steel columns $(250 \times 4.6 \mathrm{~mm}$ i.d. $)$, by Sciencehome Technology Ltd (China) with CoMetro COM 150A (USA) column packing machine. The columns were downward packed using a $40 \mathrm{MPa}$ packing press with tetrahydrofuran and 1,3-propanediol (1:3) as slurry solvent and methanol as the propelling solvent.

\section{Chromatographic conditions}

Chromatographic analysis was done on an LC3000 system purchased from Beijing Tong Heng Innovation Technology Co., Ltd. (China) assembled with an injector (Rheodyne 7725i) with $20 \mu \mathrm{L}$ sample loop, binary pumps and a UV-Vis detector (UV3000). All chromatographic data were obtained by a CXTH-3000 chromatography workstation.

Water and acetonitrile were filtered through a $0.22 \mu \mathrm{m}$ membrane filter and used as mobile phases. The UV detector was set at $280 \mathrm{~nm}$ for all the solutes. The injection volume was $10 \mu \mathrm{L}$ and the flow rate of $1 \mathrm{~mL} \mathrm{~min}{ }^{-1}$. The column temperature was at room temperature. Standard solutions were prepared as following: each compound was accurately weighed, dissolved in deionized water, diluted to $1 \mathrm{mg} \mathrm{mL}^{-1}$ and then filtered through a syringe filter $(0.22 \mu \mathrm{m})$. All the solutions were stored in the refrigerator at $4{ }^{\circ} \mathrm{C}$ and brought to room temperature before analysis. The sample solution of SHL or XBJ was injected to HPLC system after filtration through a syringe filter $(0.22 \mu \mathrm{m})$. The standard solutions and injections were analyzed under the proposed conditions in Results and Discussion section. The peaks in the chromatograph of mixtures of standard compounds or injections were assigned following the standard procedure. The column dead time was measured from the mobile phase signal in the UV detection. All of the experiments were done three times paralleled.

\section{Results and Discussion}

\section{Preparation and characterization of Mim-Sil}

The preparation procedure of Mim-Sil was modified from the literature ${ }^{35}$ for the ClPr-Sil synthesis step. We used hydrochloric acid as the catalyst instead of triethylamine because we found that it was much easier to purify the ClPr-Sil when acid was used as the catalyst. When triethylamine was used as catalyst, the elemental analysis results showed that even with extensively washing with water and ethanol, trace triethylamine was still absorbed on the silica.

$\mathrm{ClPr}-\mathrm{Sil}$ and Mim-Sil prepared in this work were characterized with elemental analysis. The data proved that methylimidazole molecules were successfully bonded onto the silica surface. Furthermore, we did elemental analysis for three batches materials and found the bonding density was repeatable (date difference was less than $0.4 \%$ ) under the same condition. Elemental analysis results of ClPr-Sil and Mim-Sil with hydrochloric acid as catalyst are shown in Table 1. The bonding density was calculated by the following equations ( $\mathrm{S}$ is the specific surface area of the silica support): ${ }^{36}$

$$
\begin{aligned}
& \operatorname{ClPr-Sil~}\left(\mu \mathrm{mol} \mathrm{m}^{-2}\right)=\frac{\mathrm{C} \%}{36 \times(1-\mathrm{C} \%-\mathrm{H} \%) \times \mathrm{S}} \\
& \operatorname{Mim}-\mathrm{Sil}\left(\mu \mathrm{mol} \mathrm{m}^{-2}\right)=\frac{\mathrm{N} \%}{28 \times(1-\mathrm{C} \%-\mathrm{H} \%-\mathrm{N} \%) \times \mathrm{S}}
\end{aligned}
$$


Table 1. Elemental analysis results of ClPr-Sil and Mim-Sil with hydrochloric acid as catalyst

\begin{tabular}{ccccc}
\hline & N / \% & C / \% & H / \% & $\begin{array}{c}\text { Coverage / } \\
\left(\mu \mathrm{mol} \mathrm{m} \mathrm{m}^{-2}\right.\end{array}$ \\
\hline \multirow{3}{*}{ ClPr-Sil } & 0.00 & 6.30 & 1.80 & 6.35 \\
& 0.00 & 6.30 & 2.18 & 6.37 \\
& 0.00 & 6.31 & 2.10 & 6.38 \\
\hline \multirow{3}{*}{ Mim-Sil } & 2.23 & 6.68 & 1.41 & 2.89 \\
& 2.21 & 6.91 & 1.81 & 2.88 \\
& 2.22 & 6.95 & 1.78 & 2.90 \\
\hline
\end{tabular}

Solid-state nuclear magnetic resonance (NMR) spectroscopy was applied to characterize the composition of chemically modified surfaces. The solid-state ${ }^{13} \mathrm{C}$ NMR spectrum for Mim-Sil was acquired as shown in Supplementary Information section Figure S1. Two important signals related to the imidazolium group bonded on the modified silica were observed at 124 and $137 \mathrm{ppm}$. Signals attributable to the carbon of methyl group could also be identified from the spectrum at $36 \mathrm{ppm}$.

Infrared spectroscopy was also used to characterize ClPr-Sil and Mim-Sil. The spectra are shown in Figure S2. When the spectra of ClPr-Sil and Mim-Sil were compared, an important band set at $1654 \mathrm{~cm}^{-1}$ which was attributed to the characteristic frequencies of imidazolium groups can be found in spectrum of Mim-Sil.

The thermogravimetric curves for ClPr-Sil and Mim-Sil can give information on their thermal stability. The ClPr-Sil silica presented no distinct mass loss below $200{ }^{\circ} \mathrm{C}$, while the Mim-Sil silica presented no distinct mass loss below $220^{\circ} \mathrm{C}$.

\section{Column packing}

In this work, it was found that the choices of slurry solvent and repellent solvent were important factors for the quality control of the prepared columns. Several kinds of common slurry and repellent solvents pairs were explored, for example, chloroform and methanol or chloroform and hexane, but the columns prepared with these solvent pairs collapsed only after several times of using. We postulated that due to special density of ionic liquid stationary phase, slurry solvent with matched density and viscosity should be chosen. Better results can be obtained when tetrahydrofuran and 1,3-propanediol (1:3) were used as slurry solvent and methanol was used as repellent solvent.

\section{Chromatographic analysis of SHL injection}

Seven standard substances which are possible allergenic constituents in SHL injections were analyzed with the Mim-Sil column. The structures of the seven compounds are shown in Figure 1. In order to provide both a practical guide to the selection of conditions to achieve a suitable separation of these seven compounds and also theoretical data analysis on the separation mode of IL column, the relationship of retention time and mobile phase constituents were studied in detail in three steps. Step one, compounds I-VII were studied one by one; then they were studied in two groups, acids group (I-V) and glycosides group (VI-VII); in the third step the separation of the seven compounds in SHL injection were studied.

Plots of variation of the retention factor $(\log k)$ of compounds I-VII against the water content $\left(\mathrm{C}_{\mathrm{B}}\right)$ in the<smiles>O=C(O)/C=C/c1ccc(O)c(O)c1</smiles>

Caffeic acid (I) Chlorogenic acid (II) Cryptochlorogenic acid (III) Neochlorogenic acid (IV)<smiles>O=C(C=Cc1ccc(O)c(O)c1)OC1C(=CC=Cc2ccc(O)c(O)c2)OC2C(C(=O)O)C(O)C2C1O</smiles>

Isochlorogenic acid $\mathrm{C}(\mathrm{V})$

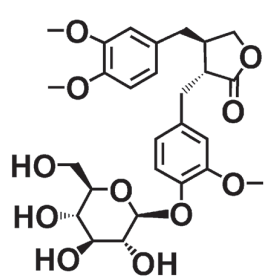

Arctiin (VI)<smiles>O=C(O)[C@H]1O[C@@H](Oc2cc3oc(-c4ccccc4)cc(=O)c3c(O)c2O)[C@H](O)[C@H](O)[C@H]1O</smiles>

Baicalin (VII)

Figure 1. The structures of the seven allergenic components in SHL injection. 
mobile phase are shown in Figure 2. It was found that retention factors did not present a monotone change $v s$. the change of the constituents of mobile phase. Instead, they showed a U-shaped relationship against changing of $C_{B}$ over the range of $0-100 \%$. And with a further look, it was found that retention factors decreased with the increase of water content when $\mathrm{C}_{\mathrm{B}}$ was over the range of $0-50 \%$ which can be recognized as a HILIC mode. The retention mechanism in HILIC is supposed to involve partitioning of analytes between a mostly organic mobile phase and a water layer at the surface of the stationary phase. ${ }^{37,38}$ Mechanism also accepted in the literature is the interaction of polar groups with water molecules from mobile phase, giving an "empirical wall" of water molecules near the silica surface. ${ }^{39}$ Furthermore, a recent study ${ }^{40}$ concluded by molecular dynamics that the ratio between the local water mole fraction at and near the surface and the nominal water mole fraction grew nonlinearly with the decrease of water content in the mobile phase. Our result is consistent with the above literature and also it confirms that water is a stronger eluting mobile phase with HILIC mode. It may also be found that retention factors increased with the increase of water content when $C_{B}$ was over the range of 50-100\% which can be recognized as an RPLC mode of hydrophobic mechanism. ${ }^{14,15}$ So in RPLC mode, water becomes weaker eluting mobile phase.

When Figures $2 \mathrm{a}$ and $2 \mathrm{~b}$ were compared, it can be found that with the variation of $C_{B}$, although the overall variation trends of retention factor for acids and glycosides were similar, the regions where dramatic change of retention factor appeared were different. For the plots of acids, range of $0-50 \% \mathrm{C}_{\mathrm{B}}$ had a large slope with the retention dropping fast when $C_{B}$ increased; whereas in the plots of glycosides the retention increased steeply at $C_{B}$ of $50-100 \%$. This kind of difference may be caused by the difference of their hydrophilicity. The range where retention factors respond sensitively should be chosen for optimizing separation conditions.

The results of step one showed the differences in separation modes of acids and glycosides, so in step two compounds I-VII were analyzed in two groups, acids group (I-V) and glycosides group (VI-VII). The chromatographic conditions were optimized in the range of $0-50 \% \mathrm{C}_{\mathrm{B}}$ for acids and $50-100 \% \mathrm{C}_{\mathrm{B}}$ for the glycosides. Figures $3 \mathrm{a}$ and $3 \mathrm{~b}$ illustrate the optimized chromatograms of acids and glycosides, respectively. It is shown that the acids mixture are well separated on the column at a flow rate of $1 \mathrm{~mL} \mathrm{~min}{ }^{-1}$, using acetonitrile (A) and $\mathrm{H}_{3} \mathrm{PO}_{4} 0.1 \%$ (B) as mobile phase. The gradient is as follows: $0-10 \mathrm{~min}, 8-10 \%$ B; 10-16 min, 10-12\% B. The glycosides mixture are well separated on the column at a flow rate of $1 \mathrm{~mL} \mathrm{~min}^{-1}$, using acetonitrile (A) and $\mathrm{H}_{3} \mathrm{PO}_{4} 0.1 \%(\mathrm{~B})$ as mobile phase. The gradient is as following: 0-3 $\mathrm{min}, 90 \% \mathrm{~B} ; 3-5 \mathrm{~min}, 90-80 \%$ B; 5-10 min, 80-70\% B; 10-15 min, 70-60\% B; 15-20 min, $60-50 \% \mathrm{~B} ; 20-25 \mathrm{~min}$ kept at $50 \% \mathrm{~B}$. During the study of acids group, we also found that the diastereoisomers like chlorogenic acid (II), cryptochlorogenic acid (III) and neochlorogenic acid (IV) can be separated on the ionic liquid based column with isocratic elution within HILIC range at $95 \%$ B. Chromatogram is shown in Figure 3c. The cis- and trans- isomers of caffeic acid can also be separated with this column, which is shown in Figure 3a with a large peak for the trans-isomer and a small peak for the $c i s$-isomer.

In the third step, SHL injection was analyzed under HILIC condition and compounds I-VII were identified simultaneously. The chromatogram is shown in Figure 4.
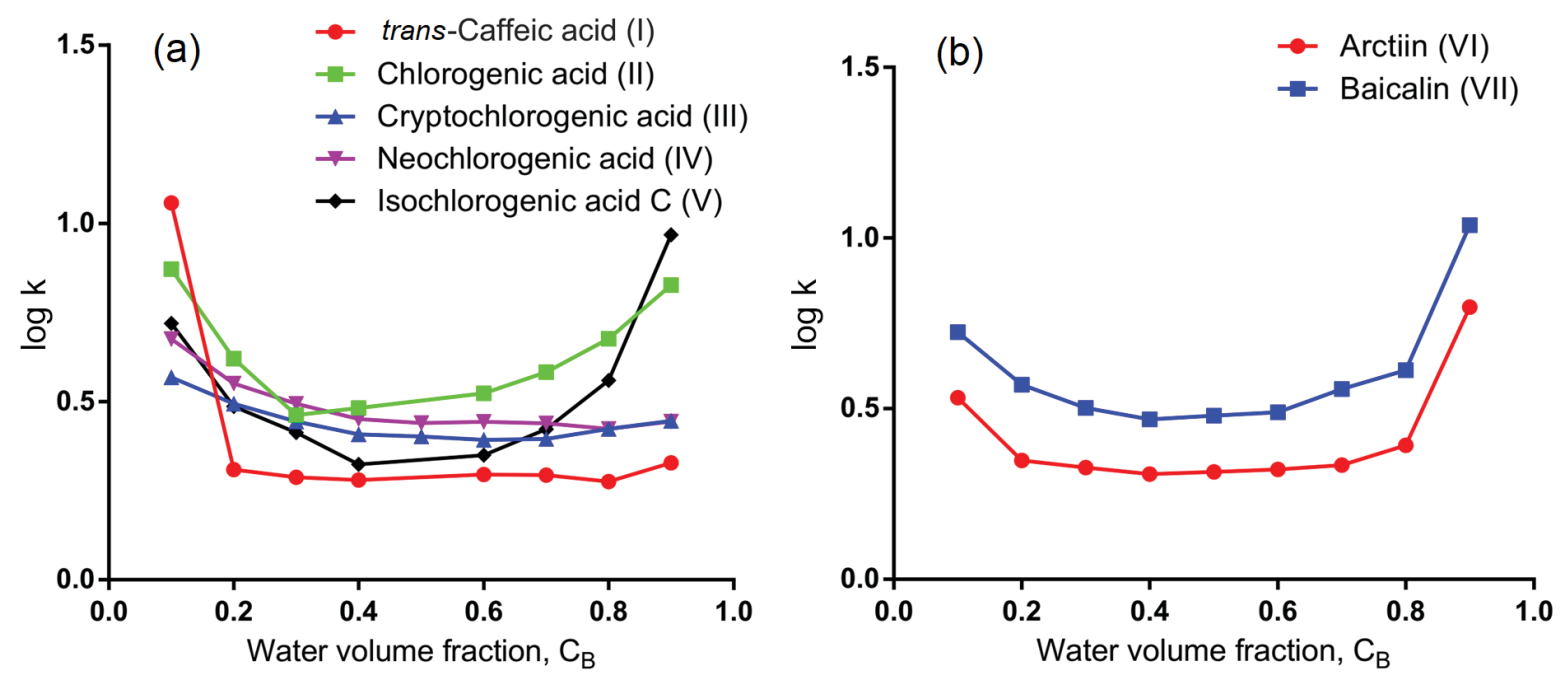

Figure 2. Plots of variation of the retention factor of compounds I-VII against the water content. Compounds I-V (a) and VI-VII (b). 

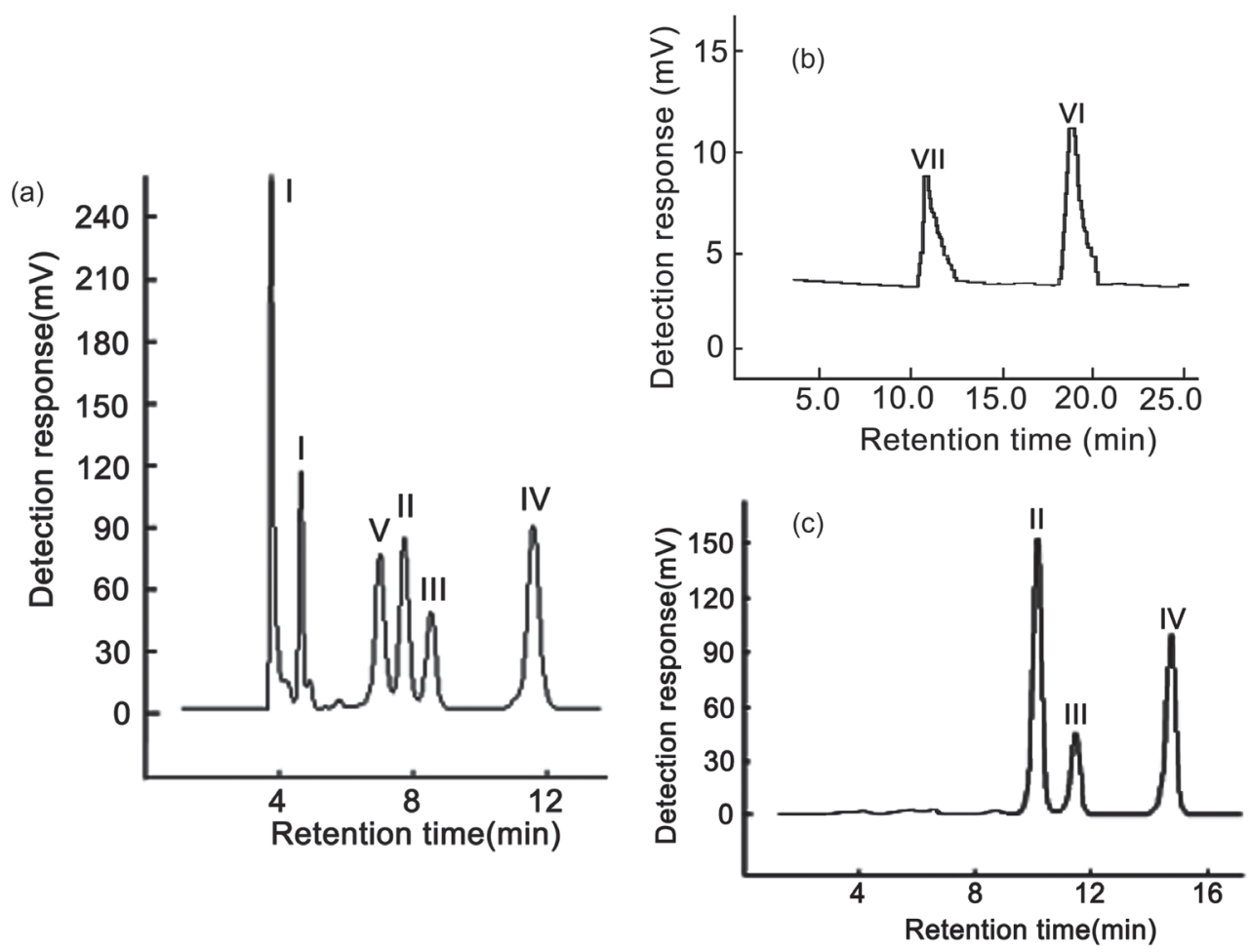

Figure 3. Optimized chromatograms of acids (a), glycosides (b) and diastereoisomers mixture (c). Caffeic acid (I), chlorogenic acid (II), cryptochlorogenic acid (III), neochlorogenic acid (IV), isocryptochlorogenic acid (V), arctiin (VI) and baicalin (VII).

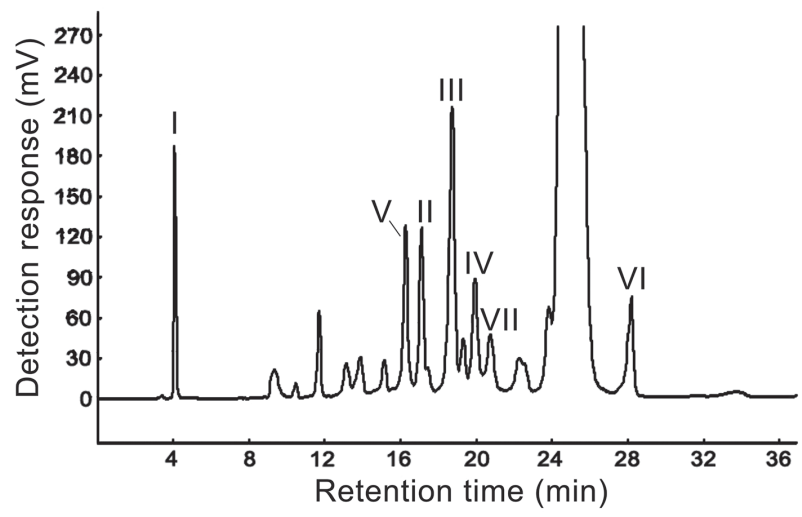

Figure 4. Optimized chromatogram of SHL injection.

\section{Chromatographic analysis of XBJ injection}

We explored the conditions for separation of eight standard substances which are bioactive components found in XBJ injections in three steps. The structures of the eight compounds are shown in Figure 5.

In step one, compounds 1-8 were classified into three groups based on the data from literature ${ }^{41}$ of $\mathrm{C}_{18}$ column. When these compounds were separated on the $\mathrm{C}_{18}$ columns, compounds 1-3 had the shortest retention time, compounds 4-6 had the medium length of retention time and compounds 7-8 had the longest retention time. Based on the reversed phase retention mode, shorter retention time means higher polarity. So compounds $\mathbf{1 - 3}$ have the relatively higher polarity, compounds 4-6 have medium polarity and compounds 7-8 have relatively lower polarity. For these three groups of compounds, we also checked the relationship between the retention time and constituents of mobile phase. For each group, different mobile phase conditions were applied to optimize the chromatographic separation. The mobile phase consisted of acetonitrile (A) and $0.1 \%$ aqueous phosphoric acid (B). The corresponding information is shown in Tables S1-S6. Figure 6 shows the results when better degree of separation and theoretical plates were obtained. In higher polarity group, better result was obtained with HILIC condition at $13 \%$ B. In medium polarity group, better result was obtained with RPLC condition at $75 \%$ B. In lower polarity group, better result was obtained with NPLC condition at $30 \%$ B. Compared with reported HILIC and RPLC columns, the IL column prepared in this report possesses multiple interactions, such as hydrogen bonding, dipolar, $\pi-\pi$, electrostatic, dispersion force and possible steric interactions. These interactions are significant for the mixed separation mode of the column under different condition with different analytes. It can also be seen that different selectivity and reversed elution orders are displayed when the constituents of the mobile phase were changed. Therefore, simply by changing the constituents of the mobile phase, IL columns may not only display selectivity and effective retention to hydrophilic compounds but also to compounds 
<smiles>O=C(O)c1cc(O)c(O)c(O)c1</smiles>

Gallic acid (1)<smiles>O=C1C=CN([C@@H]2O[C@H](CO)[C@@H](O)[C@H]2O)C1=O</smiles>

Uridine (2)<smiles>NC1=NC2C(N=CN2[C@H]2O[C@H](CO)[C@@H](O)[C@H]2O)C(=O)N1</smiles>

Guanosine (3)

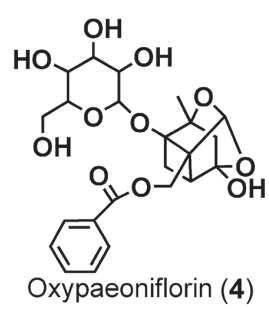<smiles>COc1cc(/C=C/C(=O)O)ccc1O</smiles>

Ferulic acid (5)<smiles>O=C(O)C(O)Cc1ccc(O)c(O)c1</smiles>

Salvianic acid A sodium (6)<smiles>O=C(C=Cc1ccc(O)c(O)c1)OC(=O)C(=O)OC(Cc1ccc(O)c(Cl)c1)C(=O)O</smiles>

Salvianolic acid B (7)

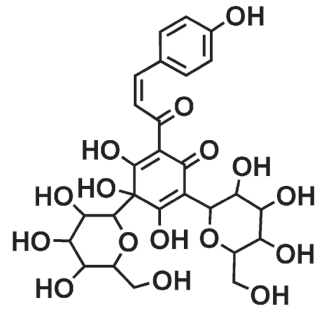

Safflor yellow A (8)

Figure 5. Structures of the eight bioactive compounds in XBJ injection.

without hydrophilic substituents. Furthermore, the result of lower polarity group presented only slight difference against the change of the constituents of mobile phase, which might be because in this situation the steric effect rather than polarity of the analytes played the main role.

In step two, mixture of compounds 1-8 was analyzed with the column. Higher degree of separation was obtained with the conditions shown in Table 2. The chromatogram is shown in Figure 7. By comparing different eluting gradient, it was found that under HILIC mode, Mim-Sil column exhibited effective retention and selectivity to these hydrophilic solutes with symmetrical peak shape.
Chromatography data of compounds 1-8 under optimized conditions are shown in Table S7.

At last, XBJ injection was analyzed with HILIC condition. Compounds 1-8 were identified simultaneously. The chromatograph is shown in Figure 8.

From the results, we can see that both SHL and XBJ injections can be separated by IL-grafted phase with mixed-mode HPLC method, although there have been some fine differences between the results due to the differences in the detection method and equipment. It may be concluded that IL-grafted phase is feasible and cost-effective way for the separation of TCM formulations.
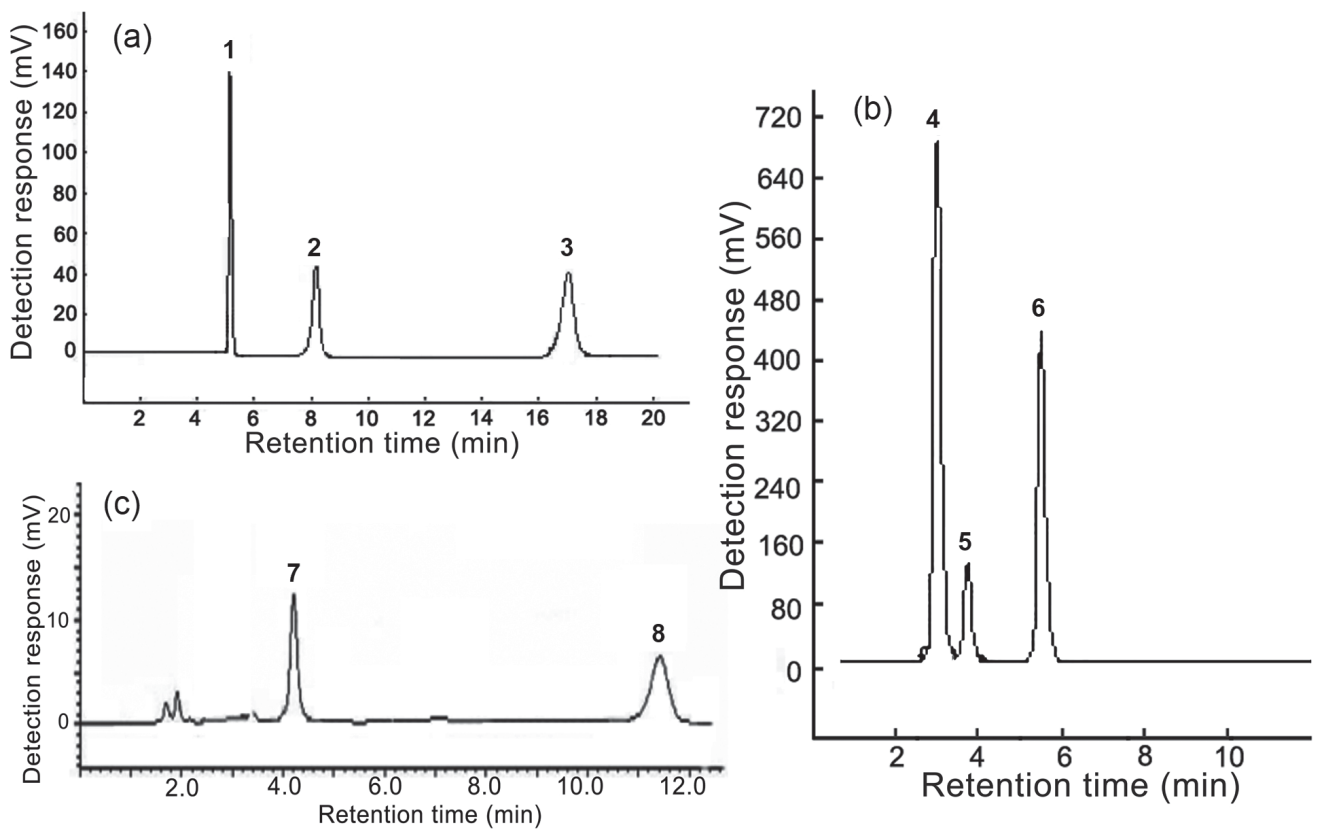

Figure 6. Chromatograms of three groups: higher polarity group (a), medium polarity group (b) and lower polarity group (c). Gallic acid (1), uridine (2), guanosine (3), oxypaeoniflorin (4), ferulic acid (5), salvianic acid A sodium (6), salvianolic acid B (7) and safflor yellow A (8). 
Table 2. Optimized eluting gradient for the mixture of compounds 1-8 on Mim-Sil column

\begin{tabular}{lcccccccc}
\hline time $/ \min$ & 0 & 15 & 28 & 40 & 45 & 48 & 52 & 58 \\
\hline A (acetonitrile) / \% & 100 & 95 & 92 & 87.5 & 80 & 79 & 78 & 95 \\
$\mathrm{~B}(0.1 \%$ aqueous phosphoric acid) / $\%$ & 0 & 5 & 8 & 12.5 & 20 & 21 & 22 & 5 \\
\hline
\end{tabular}

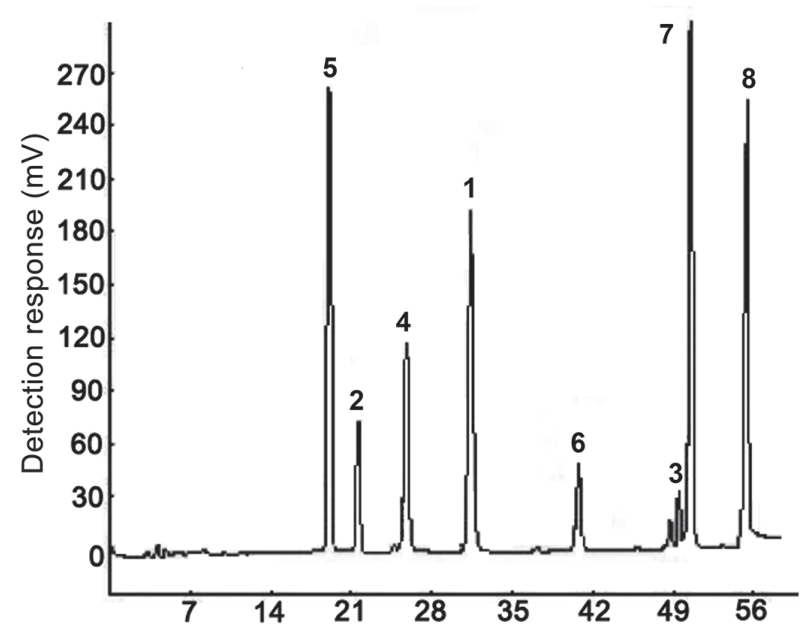

Figure 7. Chromatogram of mixture of compounds 1-8.

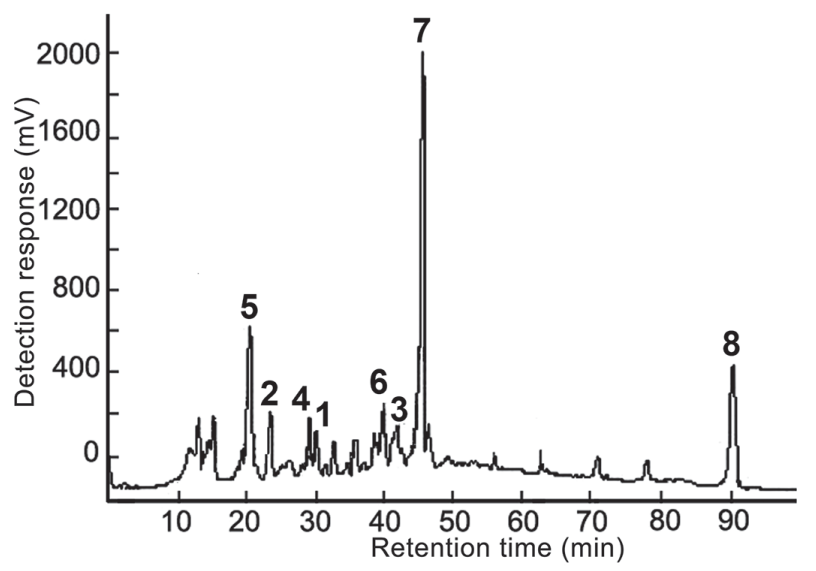

Figure 8. The optimized chromatogram of XBJ injection.

\section{Conclusions}

In summary, we established simple and effective methods to analyze and separate the allergenic components in SHL injection and mainly bioactive components in XBJ injection on Mim-Sil column with mixed-mode HPLC. For SHL injection, retention factors $v s$. mobile phase constitutions of seven allergenic components were analyzed one by one and U-shaped plots were obtained. Based on this result, eluting conditions were optimized and acids group was analyzed with HILIC mode, while glycosides group was analyzed with RPLC mode. Then SHL injection was analyzed and seven compounds were simultaneously determined. For XBJ injection, eight bioactive compounds were classified into three groups according to the polarity and their chromatographic separation conditions were optimized. Higher, medium and lower polarity groups were analyzed with HILIC, RPLC and NPLC modes, respectively. Then the mixture of these eight standard compounds was separated. At last, XBJ injection was analyzed and eight compounds were successfully simultaneously identified. The results demonstrate that mixed mode HPLC based on ionic liquid column is suitable and significant for the separation of complicated systems such as TCM injections.

\section{Supplementary Information}

Supplementary information is available free of charge at http://jbcs.sbq.org.br as PDF file.

\section{Acknowledgments}

This work was supported by Natural Science Foundation of Tianjin, China (No. 13JCYBJC38400).

\section{References}

1. Hosbach, I.; Neeb, G.; Hager, S.; Kirchhoff, S.; Kirschbaum, B.; Anaesthesia 2003, 58, 282.

2. Zhang, Q.; Yang, F. Q.; Ge, L.; Hu, Y. J.; Xia, Z. N.; J. Sep. Sci. 2017, 40, 49.

3. Liu, L.; Jiang, W.; Zhang, L.; Li, F.; Zhang, Q.; J. Sep. Sci. 2011, 34, 1834.

4. Jiang, Y.; David, B.; Tu, P.; Barbin, Y.; Anal. Chim. Acta 2010, $657,9$.

5. Yang, M.; Sun, J.; Lu, Z.; Chen, G.; Guan, S.; Liu, X.; Jiang, B.; Ye, M.; Guo, D. A.; J. Chromatogr. A, 2009, 121, 62045.

6. Liang, X.; Jin, Y.; Wang, Y.; Jin, G.; Fu, Q.; Xiao, Y.; J. Chromatogr. A 2009, 1216, 2033.

7. Li, S. P.; Zhao, J.; Yang, B.; J. Pharm. Biomed. Anal. 2011, 55, 802.

8. Ganzera, M.; Sturm, S.; J. Pharm. Biomed. Anal. 2017, 147, 211.

9. Sun, P.; Armstrong, D. W.; Anal. Chim. Acta 2010, 661, 1.

10. Ali, I.; Suhail, M.; Sanagi, M. M.; Aboul-Enein, H. Y.; Crit. Rev. Anal. Chem. 2017, 47, 332.

11. Shi, X.; Qiao, L.; Xu, G.; J. Chromatogr. A 2015, $1420,1$. 
12. Zhang, M.; Liang, X.; Jiang, S.; Qiu, H.; TrAC, Trends Anal. Chem. 2014, 53, 60.

13. Qiu, H.; Mallik, A. K.; Takafuji, M.; Liu, X.; Jiang, S.; Ihara, H.; Anal. Chim. Acta 2012, 738, 95.

14. Qiu, H.; Takafuji, M.; Liu, X.; Jiang, S.; Ihara, H.; J. Chromatogr. A 2010, 1217, 5190.

15. Sun, M.; Feng, J.; Wang, X.; Duan, H.; Li, L.; Luo, C.; J. Sep. Sci. 2014, 37, 2153.

16. Qiao, L.; Li, H.; Shan, Y.; Wang, S.; Shi, X.; Lu, X.; Xu, G.; J. Chromatogr. A 2014, 1330, 40.

17. Liu, M.; Chen, E. X.; Ji, R.; Semin, D.; J. Chromatogr. A 2008 , $1188,255$.

18. Qiao, L.; Shi, X.; Lu, X.; Xu, G.; J. Chromatogr. A 2015, 1396, 62.

19. Zhang, L.; Dai, Q.; Qiao, X.; Yu, C.; Qin, X.; Yan, H.; TrAC, Trends Anal. Chem. 2016, 82, 143.

20. Hu, K.; Zhang, W.; Yang, H.; Cui, Y.; Zhang, J.; Zhao, W.; Yu, A.; Zhang, S.; Talanta 2016, 152, 392.

21. Yang, B.; Liu, H.; Chen, J.; Guan, M.; Qiu, H.; J. Chromatogr. A 2016, 1468, 79.

22. Chinese Pharmcopoeia Commission; Pharmacopoeia of the People's Republic of China; Chinese Medical Science and Technology Press: Beijing, 2015, vol. 1, p. 1108.

23. Zhang, H.; Chen, Q.; Zhou, W.; Gao, S.; Lin, H.; Ye, S.; Xu, Y.; Cai, J.; Evidence-Based Complementary Altern. Med. 2013, 987326.

24. Shi, J.; Cao, B.; Wang, X. W.; Aa, J. Y.; Duan, J. A.; Zhu, X. X.; Wang, G. J.; Liu, C. X.; J. Chromatogr. B: Anal. Technol. Biomed. Life Sci. 2016, 1026, 204.

25. Ye, J.; Song, X.; Liu, Z.; Zhao, X.; Geng, L.; Bi, K.; Chen, X.; J. Chromatogr. B: Anal. Technol. Biomed. Life Sci. 2012, 898, 130.

26. Han, S. L.; Zhang, T.; Huang, J.; Cui, R. H.; He, L. C.; J. Pharm. Biomed. Anal. 2014, 88, 602.
27. Sun, H.; Liu, M.; Lin, Z.; Jiang, H.; Niu, Y.; Wang, H.; Chen, S.; J. Pharm. Biomed. Anal. 2015, 115, 86.

28. Chen, L.; Hakamata, H.; Kusu, F.; Wang, Z.; Gao, H.; Kotani, A.; J. Pharm. Biomed. Anal. 2014, 95, 93.

29. Niu, Y. Q.; Ma, Y. Y.; J. Nongken. Med. 2009, 31, 451.

30. Chen, Y. X.; Li, C. S.; Chin. J. Emerg. Med. 2013, 22, $130 \mathrm{e} 5$.

31. Huang, H.; Ji, L.; Song, S.; Wang, J.; Wei, N.; Jiang, M.; Bai, G.; Luo, G.; Phytochem. Anal. 2011, 22, 330.

32. Jiang, M.; Zhou, M.; Han, Y.; Xing, L.; Zhao, H.; Dong, L.; Bai, G.; Luo, G.; J. Ethnopharmacol. 2013, 147, 426.

33. Jia, P.; Wang, S.; Meng, X.; Lan, W.; Luo, J.; Liao, S.; Xiao, C.; Zheng, X.; Li, L.; Liu, Q.; Zheng, J.; Zhou, Y.; Zheng, X.; Talanta 2013, 107, 103.

34. Zuo, L.; Sun, Z.; Hu, Y.; Sun, Y.; Xue, W.; Zhou, L.; Zhang, J.; Bao, X.; Zhu, Z.; Suo, G.; Zhang, X.; J. Pharm. Biomed. Anal. 2017, 137, 220.

35. Qiu, H.; Jiang, S.; Liu, X.; J. Chromatogr. A 2006, 1103, 265.

36. Qiu, H.; Jiang, Q.; Wei, Z.; Wang, X.; Liu, X.; Jiang, S.; J. Chromatogr. A 2007, 1163, 63.

37. Schuster, G.; Lindner, W.; J. Chromatogr. A 2013, 1273, 73.

38. Kumar, A.; Heaton, J. C.; McCalley, D. V.; J. Chromatogr. A 2013, 1276, 33.

39. Faria, A.; Collins, C. H.; Jardim, I. C. S. F.; J. Braz. Chem. Soc. 2009, 20, 1385.

40. Melnikov, S. M.; Höltzel, A.; Seidel-Morgenstern, A.; Tallarek, U.; Angew. Chem., Int. Ed. 2012, 51, 6251.

41. Huang, H.; Wang, J.; Fu, J. Z.; Wang, L. Q.; Zhao, H. Z.; Song, S. Y.; Ji, L. X.; Jiang, M.; Bai, G.; Luo, G. A.; J. Anal. Chem. 2013, 68, 348.
Submitted: September 26, 2017

Published online: January 26, 2018 